\title{
A MISSION-ENABLING UV LASER FOR MASS SPECTROMETRY (UVMS) WITH CONTINUOUSLY SELECTABLE OUTPUT FOR IN SITU PLANETARY EXPLORATION
}

\author{
D. Barry Coyle ${ }^{\mathrm{a}}$, Demetrios Poulios ${ }^{\mathrm{b}}$, Ricardo Arevalo ${ }^{\mathrm{a}}$, Greg B. Clarke ${ }^{\mathrm{b}}$, Paul R. Stysley ${ }^{\mathrm{a}}$ \\ ${ }^{a}$ NASA Goddard Space Flight Center, Lasers and Electro-Optics Branch, Greenbelt, MD 20771 \\ ${ }^{b}$ The American University, Dept. of Physics, 4400 Massachusetts Ave NW, Washington, DC 20016
}

\begin{abstract}
Ultra-compact, nanosecond-class spaceflight-compatible UV lasers are finding increasing application in laser desorption, excitation, and ionization analytical applications on planetary missions, such as the detection and characterization of potential molecular biosignatures on Mars or icy moon surfaces. A short pulsed, solid state, UV laser is under development with selectable pulse energy capabilities for optimized sample ion production at a planetary surface.
\end{abstract}

Keywords: diode pumped laser, Nd:YAG, desorption, mass spectrometer

1.

\section{INTRODUCTION}

Laser ablation and desorption microprocessing, coupled with mass spectrometric analysis, enables the in situ chemical (organic and inorganic) characterization of planetary materials with sub-mm spatial resolution. Future NASA missions to icy Jovian moons, Mars, and/or other planetary bodies will need UV laser systems tuned to specific wavelengths in order to effectively irradiate collected surface samples (including powdered fines or solid sample cores). Laser desorption methods (often referred to as "soft ablation") rely on lower fluences to liberate and ionize organic compounds without inducing excessive fragmentation of the parent molecule; the covalent bonds characteristic of organic molecules often require less energy (on the order of $\leq 0.1 \mathrm{~J} \bullet \mathrm{cm}-2$ ) to break than the bonds of many mineral crystal lattices (which require $\geq 1.0 \mathrm{~J} \bullet \mathrm{cm}-2$ ). Excessive irradiation of organic compounds can result in the degradation and atomization of those compounds, thus careful control of pulse energy deposition for sample ion production is critical. Infrared (i.e., $1064 \mathrm{~nm})$ and shallow UV wavelengths (i.e., $266 \mathrm{~nm}$ ) are commonly employed for laser desorption/ablation processing. An example of a spaceflight implementation of a laser desorption mass spectrometer is the Mars Organic Molecule Analyzer (MOMA) instrument onboard ESA's ExoMars rover. ${ }^{1}$ The MOMA laser produces $266 \mathrm{~nm}$ laser pulses, directed at targeted samples of Martian surface and subsurface materials (as deep as $2 \mathrm{~m}$ ), in order to generate enough ions to fill the linear ion trap mass analyzer.

Table 1: Fundamental operational requirements of the UVMS laser.

\begin{tabular}{|l|l|}
\hline Specification & Requirement \\
\hline Wavelength & $266 \mathrm{~nm}$ \\
\hline Pulse Energy & $>200 \mathrm{uJ}$ \\
\hline Pulse Duration & $<2.5 \mathrm{~ns}$ \\
\hline Energy Tunability & $\sim 1 \%$ increments \\
\hline Repetition Rate & $100 \mathrm{~Hz}$ \\
\hline Operation & 50 shot bursts \\
\hline
\end{tabular}


The UV Mass Spectrometry (UVMS) laser effort seeks to develop a flight-capable laser system, first with a breadboard demonstration and ultimately a compact packaged unit, that could ultimately be adapted to future landers, mobile rovers, or planetary body sample missions. To keep the ultimate application of such a laser open to a wide range of future applications, the mechanical design will be subjected to GEVS-type (general environmental verification system) in vibration and thermal vacuum testing. ${ }^{2}$ The critical performance specifications to be met are listed in Table 1.

The UVMS laser consists of three stages: a diode-pumped passively Q-switched oscillator; an electro-optic polarizationbased attenuator; and two nonlinear frequency conversion crystals (see Figure 1). Like the Mercury laser Altimeter (MLA), Lunar Orbiter Laser Altimeter (LOLA), and Geoscience Laser Altimeter System (GLAS) instruments, the UVMS oscillator utilizes Nd:YAG as the laser gain medium with a $\mathrm{Cr}^{+4}$ :YAG passive Q-switch to achieve high ( 1 MW)

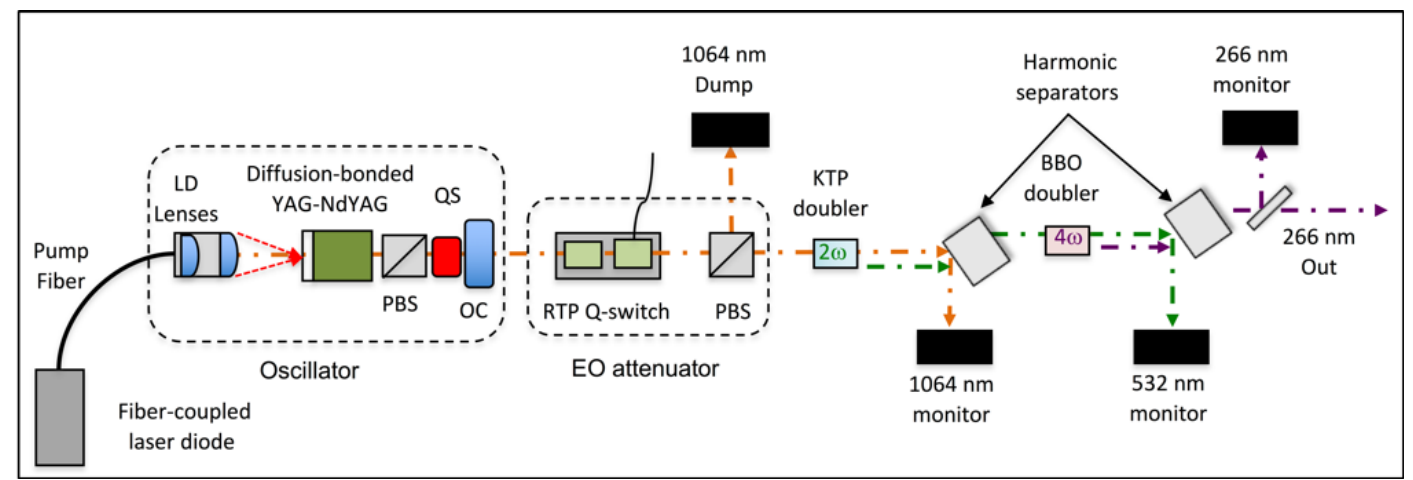

Figure 1: Initial breadboard layout of UVM laser showing oscillator, attenuator, and frequency conversion stages.

peak power output. $3,4,5$ However, these systems were required to operate in a very different environment as well as the effective duty factors and repetition rate.

The laser is operated in an end-pumped configuration with a fiber-coupled laser diode module supplying pump power through a pair of aspheric lenses. ${ }^{6}$ This arrangement allows for a compact $(<10 \mathrm{~cm})$ laser cavity, and also for the diode pump and associated control electronics to be located virtually anywhere. This layout also allows for the only sensitive thermally control item (the laser diode) to be located with the instrument electronics, where the main laser enclosure located essentially attached to the mass spectrometer structure near the sample, with minimal thermal control.

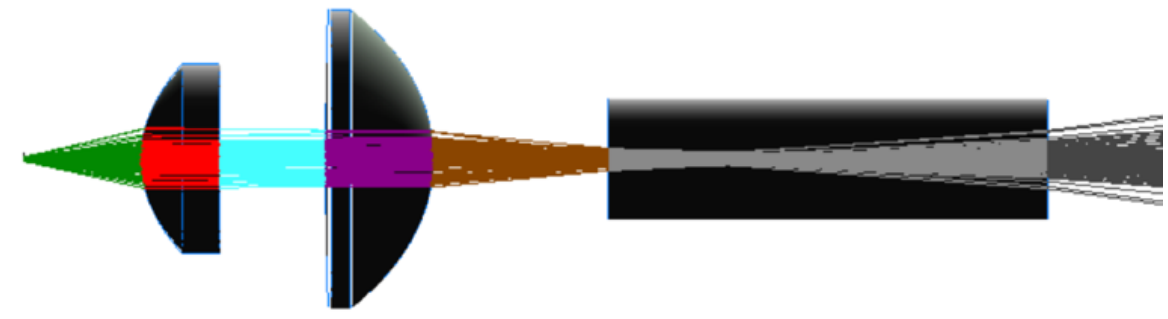

Figure 2: Raytrace of fiber-coupled pump source re-imaging system. We employ a pair of commercial aspheres to couple the 885 $\mathrm{nm}$ pump energy from a $110 \mathrm{~W}$ (rated $\mathrm{CW}$ ) diode laser and $400 \mathrm{um}$ core fiber, operated pulsed at $\sim 60 \mathrm{~W}$ per pulse.

An intra-cavity polarizing beam splitter (PBS) cube forces the laser to oscillate in a single linear polarization state in order to achieve high harmonic generation efficiency. In all, the oscillator stage produces pulse energies in excess of 1 $\mathrm{mJ}$ with short temporal pulse widths $(<3 \mathrm{~ns})$. To provide precise tuning of the $266 \mathrm{~nm}$ laser output without distorting the temporal envelope, an electro-optic attenuator consisting of an RTP Pockels cell and a PBS cube will be used to actively the $1064 \mathrm{~nm}$ power entering the nonlinear conversion stages. High voltage pulses applied to the RTP cell are synchronized to the diode pump pulse to ensure proper timing, and the HV amplitude is controls the fraction of incident light transmitted by the PBS cube. Finally, the transmitted $1064 \mathrm{~nm}$ light is frequency-doubled twice using KTP and 
BBO nonlinear crystals, respectively. The crystals are placed in series with dichroic filters removing light at the fundamental wavelength after each doubling stage; the residual fundamental light then serves as a performance monitor for each stage.

2.

\section{TRANSMITTER DESIGN - BREADBOARD}

\subsection{Cavity Design}

The design of the $1064 \mathrm{~nm} \mathrm{Nd:YAG} \mathrm{oscillator} \mathrm{was} \mathrm{driven} \mathrm{by} \mathrm{a} \mathrm{number} \mathrm{of} \mathrm{considerations,} \mathrm{including} \mathrm{small} \mathrm{form} \mathrm{factor,}$ incorporation of passive q-switching, good beam quality for efficient nonlinear conversion, and linearly polarized output. We began the design process by choosing a nominal cavity length of $7 \mathrm{~cm}$ and a $3 \mathrm{~mm}$ diameter, $15 \mathrm{~mm}$ long $1.1 \%$ doped Nd:YAG rod as the gain medium. We chose to end-pump a small laser rod for a long depth of pump length, and the ability to remotely locate the pump source. Side pumping a small zig-zag slab with a single diode array was our other option however, inherent single mode operation would likely be more difficult where the end pumped option can be tailed to mode match the cavity TEM00 mode with greater precision. One end of the YAG rod was coated for high reflection of $1064 \mathrm{~nm}$ light (and high transmission of pump light) so that it functioned as a cavity mirror. We then modeled the laser cavity using a $150 \mathrm{~cm}$ concave output coupler, resulting in a $\sim 650 \mathrm{um}$ beam in the gain medium. We then used raytrace software to model coupling of pump light from a $100 \mathrm{~W}, 885 \mathrm{~nm}$ fiber-coupled diode laser with a 400 um core and $0.22 \mathrm{NA}$ delivery fiber. We used $885 \mathrm{~nm}$ as a pump after considering the burst mode operational requirement, and the end-pumped advantage of our gain medium. By moving from the more common $808 \mathrm{~nm}$ pump absorption line to $885 \mathrm{~nm}$, the quantum defect heating will be measurable less, thus producing less thermo-optic effects.

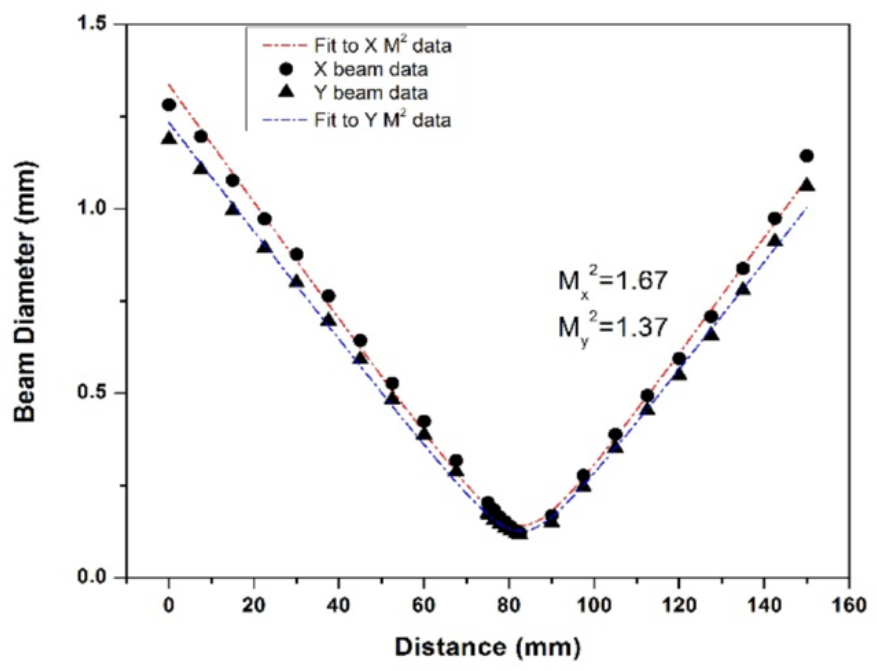

Figure 3: $\mathrm{M}^{2}$ fits of depth-of-focus curves in $\mathrm{x}$ - and $\mathrm{y}$-axis for output coupler. The small cavity and excellent mode-matching of pump volume to the cavity's inherent TEM00 volume helps to keep the spatial noise to a minimum and excellent final beam quality.

The Nd:YAG $885 \mathrm{~nm}$ absorption line is less than $2.5 \mathrm{~nm}$ in spectral width (FWHM), and the maximum peak absorption is around $1.8 \mathrm{~cm}^{-1}$ for a $1.0 \% \mathrm{Nd}$ doped crystal; about $5 \mathrm{X}$ less than that of $808 \mathrm{~nm} .{ }^{7}$ Thus, the depth of focus should further reduce thermal lensing effects in the crystal, making for a cleaner TEM00 beam, and less pulse to pulse variability over burst mode operation. A pair of aspheric lenses were used to collimate and refocus the light into the YAG rod. Using an absorption coefficient of $2.5 \mathrm{~cm}^{-1}$, we found that using off-the-shelf aspheric optics with $6.25 \mathrm{~mm}$ (collimating) and $15.0 \mathrm{~mm}$ (coupling) focal lengths spaced appropriately yielded a good mode match with the projected 650 um diameter laser beam.

Using this modeling data as a guide, we constructed the breadboard version and characterized its performance. In the initial version, we attempted to use a (111)-cut Cr:YAG passive q-switch to polarize the output without an internal PBS 
cube. However, it was found that the output tended to be unstable, and so we installed a $5 \mathrm{~mm}$ AR-coated PBS cube in the cavity. We experimented with a range of passive Q-switch optical densities (ODs) between 0.6 and 0.9 , output coupler curvatures and reflectivities. It was found that the 0.8 switch in combination with a $40 \%$ reflective, $150 \mathrm{~cm}$ concave output coupler yielded the most stable output between 1.0-1.1 mJ in a $\sim 3 \mathrm{~ns}$ pulse for this $10 \mathrm{~cm}$ cavity. The buildup time (ie, amount of time for the Q-switched pulse to evolve after pumping begins) was $\sim 350$ us. We then measured the beam quality of the output by sampling a small portion of the laser output, directing it through a spherical lens, and measuring the beam diameter as a function of distance. The resulting curves were then fit with Gaussian beam propagation functions in order to determine the $\mathrm{M}^{2}$ values in $\mathrm{x}$ and $\mathrm{y}$ (a perfect Gaussian beam has an $\mathrm{M}^{2}$ of 1.0 ; in general, anything below 2 is considered to be good beam quality). The measured values found from the curve fits are $\mathrm{M}_{\mathrm{x}}{ }^{2}=1.67$ and $\mathrm{M}_{\mathrm{y}}{ }^{2}=1.37$. These values deviate from the ideal value of 1.0 because of imperfect modeling of the pump coupling and thermal effects, but are of good enough quality to provide a high nonlinear conversion efficiency to 532 nm.

\subsection{Electro-Optic Attenuation}

Due to the steady state, non-tunable nature of passive Q-switching, we chose to use external methods of attenuating the output pulses prior to frequency conversion. The EO attenuation stage operates by first converting linearly polarized light from the Nd:YAG laser to elliptical polarization via phase retardation in an RTP Pockels cell, and subsequently removing the s-polarized component of the transmitted light by a PBS cube. In this manner, the amount of light passing the PBS can be precisely tuned between $0-100 \%$ simply by varying the applied voltage to the RTP cell.



Figure 4: Normalized power rejected from optical train from post-RTP polarizing beam splitter (black curve) vs the applied RTP voltage. Theoretical values are shown in red.

RTP was chosen for the EO attenuator because of its low switching voltage $(\sim 2 \mathrm{kV}$ for half-wave retardation of 1064 $\mathrm{nm}$ ) relative to crystals such as $\mathrm{BBO}$ and $\mathrm{KD} * \mathrm{P}$, and also because it is not susceptible to piezoelectric ringing effects that tend to modulate the transmitted light. The RTP crystals are aligned such that the polarization of the incoming light is $45^{\circ}$ to the fast and slow axes of the crystals. The small size of the unit helps quite a bit as well, as the overall focus of this effort is mass reduction and minimum footprint. An additional advantage is that RTP has been qualified for space flight use via the ICEsat2 mission as the laser cavity's electro-topical Q-switch. This material (and this specific make and model) has been thoroughly vetted through extensive environmental testing and thus, with careful attention to detail, this RTP unit will perform well for our comparative low impact use as an energy attenuator at a distant planetary body. ${ }^{8}$

\subsection{Frequency Conversion}

The frequency conversion of the fundamental wavelength $1064 \mathrm{~nm}$ to $266 \mathrm{~nm}$ is carried out in two stages. For the second harmonic conversion of $1064 \mathrm{~nm}$ to $532 \mathrm{~nm}$, a commercially available $3 \times 3 \times 6 \mathrm{~mm}^{3} \mathrm{KTP}$ crystal was chosen. KTP 
was selected over LBO because of its wide temperature bandwidth and high conversion efficiency at low to intermediate peak powers. A comprehensive study of conversion efficiency to $532 \mathrm{~nm}$ was conducted using SNLO software with inputs derived from the modeling results of the Nd:YAG laser. To test theoretical predictions of doubling performance, the KTP crystal was placed after the Nd:YAG laser and EO attenuator and aligned for maximum conversion to $532 \mathrm{~nm}$. A peak efficiency of nearly $70 \%$ was achieved, which is slightly higher than the value predicted by SNLO. The beam quality of the $532 \mathrm{~nm}$ light was then tested by placing a pickoff wedge in the beam path to sample a small fraction of the beam, after which the sampled beam diameter is measured as a function of distance after passing through a spherical

lens. However, to reduce total system part count for final compactness and light weight, we found we could achieve over $50 \%$ conversion to $532 \mathrm{~nm}$ simply placing the KTP after the RTP. We later installed a $1 / 2$ wave plate to optimize the polarization in lieu of rotation the KTP for easier alignment.

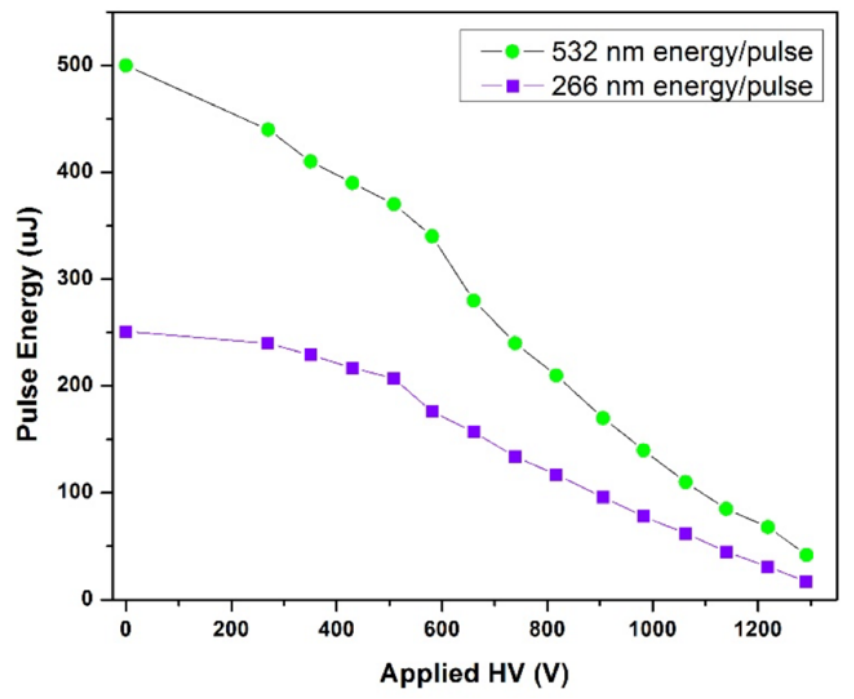

Figure 5: The $266 \mathrm{~nm}$ (violet) and $532 \mathrm{~nm}$ (green) pulse energies are plotted as a function of RTP attenuator voltage. The final UV pulse can be tuned to virtually any value between 0-250 uJ, by applied a 0-5 V DC signal to the HV driver.

For creating the fourth harmonic generation to $266 \mathrm{~nm}$, a commercial BBO offering was selected, and we simply frequency doubled $532 \mathrm{~nm}$. The remaining off-axis $1064 \mathrm{~nm}$ energy was dumped or sent out of harm's way. This was a fairly easy choice as few materials have the necessary transparency and phase-matching ability in the deep UV, but it should be noted that BBO offers a similar advantage to KTP in its wide temperature acceptance bandwidth, high damage threshold, and relatively low alignment sensitivity for "reasonable" conversion. A 4x4x6 mm 3 BBO crystal was mounted in an aluminum heat sink and placed in series with the KTP crystal; no active temperature control was employed. A harmonic separator was placed between the two nonlinear crystals to remove residual $1064 \mathrm{~nm}$ light from the KTP frequency doubler. When properly aligned, the laser produced $\sim 250 \mathrm{uJ}$ of $266 \mathrm{~nm}$ light, representing an overall conversion efficiency of 23-24\%. We then measured the $532 \mathrm{~nm}$ and $266 \mathrm{~nm}$ pulse energies over a number of discrete attenuator voltages. As shown in Figure 6, the $266 \mathrm{~nm}$ pulse energy was readily tuned between $250 \mathrm{uJ}$ and $<10 \mathrm{uJ}$ over the an RTP applied voltage range $0-1300 \mathrm{~V}$. With the appropriate HV driver, we were able to simply apply an external voltage between $0-5 \mathrm{~V}$, easily reproduced in a remote instrument controller, to dial in the exact pulse energy. Furthermore, this energy selection can be performed on a shot to shot basis, depending on the final science requirements and sample parameters.

3.

HIGH TRL PACKAGING

\subsection{Packaged Transmitter Assembly}

Once the breadboard was thoroughly tested, we begun a packaging effort to study how far we could minimize the footprint and mass to achieve the essential requirements from Table 1. Furthermore, the operational parameters have adapted to include burst mode operation for a sample analysis mission, similar to the Mars MOMA instrument, where the 


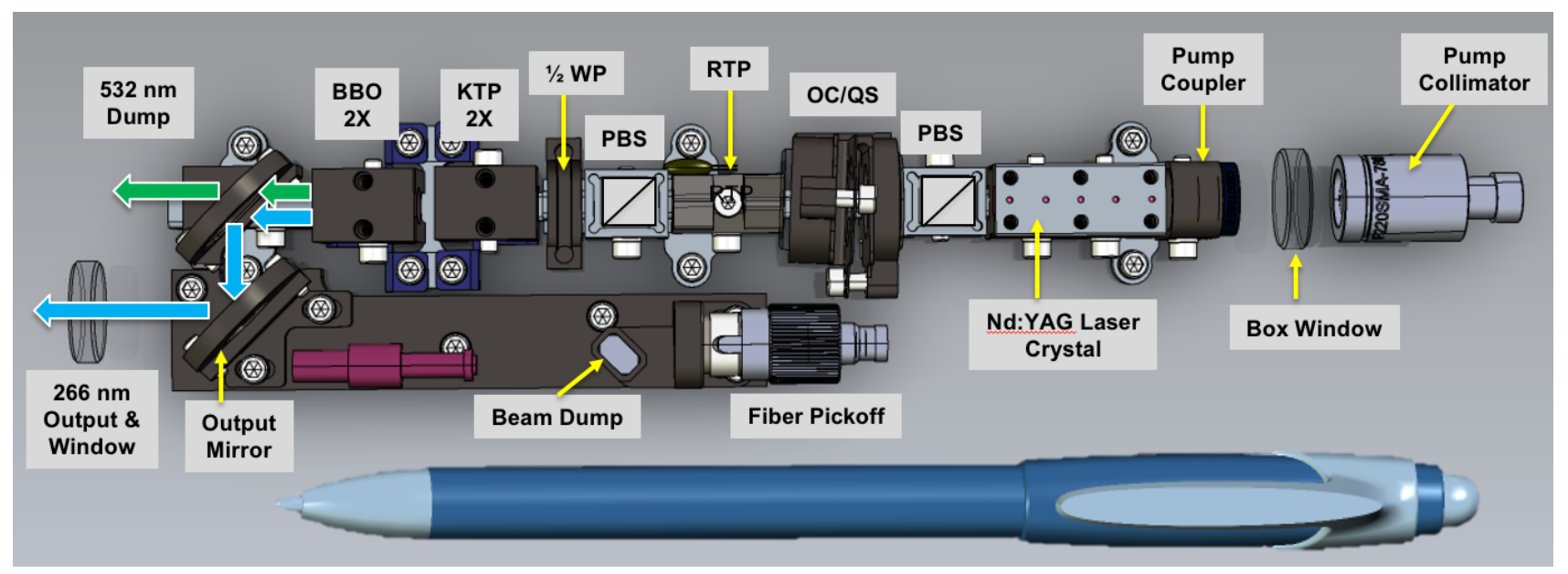

Figure 6: Interior layout of our packaging study for minimum size, mass, and footprint. We have focused on only using commercially available, yet high TRL components. The RTP and PBS act as a $1064 \mathrm{~nm}$ energy "throttle" to adjust the final $266 \mathrm{~nm}$ energy production. Note; the pen is shown only for scale.

on-board ion trap mass spectrometer requires pulses, or busts of ions to be fed into the vacuum chamber. We will operate the packaged unit at $100 \mathrm{~Hz}$ repetition rate, but in50 shot bursts of up to $2 \mathrm{sec}$ intervals. We are using an all aluminum housing, with titanium optical mounts for high strength, low mass, and an improved coefficient of thermal expansion (CTE) match with the optical materials. When installed in an all-aluminum, pressurized enclosure, we will have a high TRL (Technology Readiness Level) design ready for GEVS environmental testing by early summer 2018 .

With the use of custom optic mounts, and transferring our demonstration breadboard to our high TRL design, each piece was tailored to its optic size, and manufactured out of Titanium. This allowed the Q-switched laser cavity to shrink to



Figure 7: The UVMS laser nearing completion in its final design, shown in Figure 6. The enclosure is to the left, with a hermetic, welded micro-D connection for all electrical. The pump fiber and asphere focusing optics are in the lower center. Minimal heat removal is needed due to the low duty cycle, and the fiber-coupled laser diode pump source will be located elsewhere with dedicated, or shared, thermo-electrics with other electronics, depending on the instrument's thermal requirements and final mission destination. 
$6 \mathrm{~cm}$, which reduced the Q-switched pulse width to $2.4 \mathrm{~ns}$, but the cavity stability became more sensitive. Effectively a $40 \%$ reduction in cavity length is significant with a relatively long output coupler end mirror of $150 \mathrm{~cm}$. We found a move to a $50 \mathrm{~cm}$ radius of curvature output coupler produced the best cavity stability and still maintained performance. By calculating the cavity stability using the standard g1, g2 method, we find the breadboard was not far from the planeparallel asymptote with a $\mathrm{g} 1=1-(\mathrm{L} / \mathrm{R} 1)$ and, $\mathrm{g} 2=1-(\mathrm{L} / \mathrm{R} 2)$, or: $\mathrm{g} 1 \mathrm{~g} 2=0.93$. The greatest stability lies near the middle of the range: $0 \leq \mathrm{g} 1 \mathrm{~g} 2 \leq 1$. The cavity length reduction becomes: $\mathrm{g} 1 \mathrm{~g} 2=0.96$. This move seems small, but "feels" significant under alignment conditions. By moving the output coupler to a $50 \mathrm{~cm}$ ROC, then: g1g2=0.88, and thus greater range of mechanical motion for TEM00 operation.

4.

\section{CONCLUSION}

The packaged, high TRL version of the UVMS laser is under assembly, and has readily produced $>0.5 \mathrm{~mJ}$ of $532 \mathrm{~nm}$ and $>200 \mathrm{uJ}$ at $266 \mathrm{~nm}$, with excellent beam quality. As the final sealed assembly is not yet complete at the time of this submission, we fully expect the packaged UVMS laser to perform as well or better than the breadboard. Once the remaining optics have been bonded in place, all fasteners torqued to specification, and fiber optics installed, the box will have interior harnessing and fiber run throughout with careful strain relief and bend radii where needed. The enclosure should adequately shield the outside world from the HV electro magnetic interference noise generated by the HV driver. Once sealed, purged, and pressurized with ulta-pure dry air, the high-TRL UVMS laser will be sent though GEVS level vibration testing and characterized.
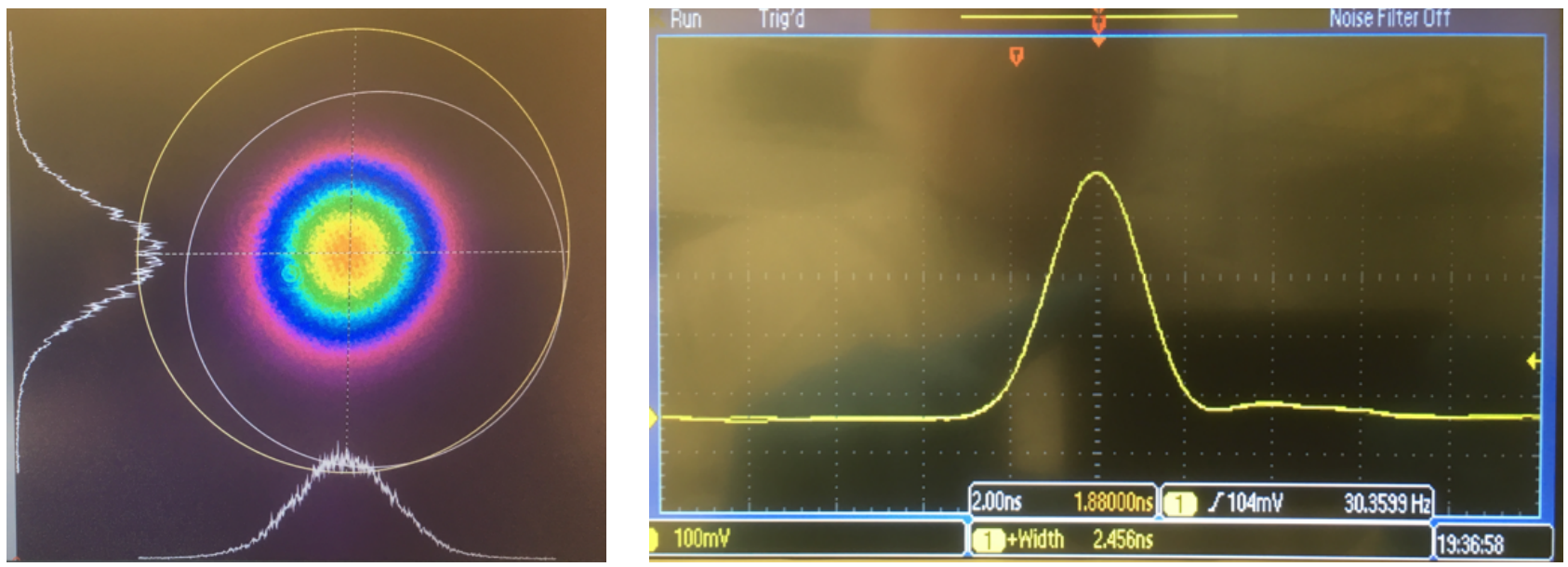

Figure 8 \& 9: TEM00 output of the packaged UVMS laser, $1.1 \mathrm{~mJ}$ output, with new $50 \mathrm{~cm}$ output coupler and shorter cavity. The final QS pulse width is $<2.5 \mathrm{~ns}$ and pump laser temperature bandwidth is $\sim \pm 2.5^{\circ} \mathrm{C}$, providing for a less overall temperature sensitive device, better suited for harsh extraterrestrial planetary surface use.

\section{REFERENCES}

1. Xiang Li, Ryan M. Danell, Veronica T. Pinnick, Andrej Grubisic, Friso van Amerom, Ricardo D. Arevalo, Stephanie A. Getty, William B. Brinckerhoff, Adrian E. Southard, Zachary D. Gonnsen, Tomoko Adachi, "Mars Organic Molecule Analyzer (MOMA) laser desorption/ionization source design and performance characterization," International Journal of Mass Spectrometry, 422, 177-187 (2017).

2. Taku Saiki et al, " $\mathrm{Nd}^{3+}$ - and $\mathrm{Cr}^{3+}$-Doped Yttrium Aluminum Garnet Ceramic Pulse Laser Using $\mathrm{Cr}^{4+}$-Doped Yttrium Aluminum Garnet Crystal Passive Q-Switch”, Japanese Journal of Applied Physics 48, (2009).

3. Danny J. Krebs, Anne-Marie Novo-Gradac, Steven X. Li, Steven J. Lindauer, Robert S. Afzal, and Anthony W. Yu, "Compact, passively Q-switched Nd:YAG laser for the MESSENGER mission to Mercury," Appl. Opt. 44, 1715-1718 (2005). 
4. H. Riris et al., "The Lunar Orbiter Laser Altimeter (LOLA) on NASA's Lunar Reconnaissance Orbiter (LRO) mission," 2009 Conference on Lasers and Electro-Optics and 2009 Conference on Quantum electronics and Laser Science Conference, Baltimore, MD, (2009).

5. R. S. Afzal et al., "The Geoscience Laser Altimeter System (GLAS) Laser Transmitter," IEEE Journal of Selected Topics in Quantum Electronics, vol. 13, no. 3, 511-536, (2007).

6. Yufei Ma, Ying He, Xin Yu, Xudong Li, Jiang Li, Renpeng Yan, Jiangbo Peng, Xinlu Zhang, Rui Sun, Yubai Pan, and Deying Chen, "Multiple-beam, pulse-burst, passively Q-switched ceramic Nd:YAG laser under microlens array pumping," Opt. Express 23, 24955-24961 (2015).

7. Make Frede, Ralf Wilhelm, and Dieter Kracht, " $250 \mathrm{~W}$ end-pumped Nd:AYG laser with direct pumping into the upper laser level," Optics Letters, 31, No. 24, 3618-3619, (2006).

8. Nicholas W. Sawruk, Patrick M. Burns, Ryan E. Edwards, Theodore Wysocki, Andre VanTuijl, Viatcheslav Litvinovitch, Edward Sullivan, Floyd E. Hovis, "ICESat-2 laser technology readiness level evolution," Proceedings Solid State Lasers XXIV: Technology and Devices; 93420 (2015). 